\title{
The Strong Disjoint Blow-Up/Collapse Property
}

\author{
Héctor N. Salas \\ Department of Mathematics, University of Puerto Rico, Mayagüez, PR 00681, USA \\ Correspondence should be addressed to Héctor N. Salas; hector.salas@upr.edu \\ Received 9 May 2013; Revised 14 August 2013; Accepted 15 August 2013 \\ Academic Editor: Ajda Fošner
}

Copyright (C) 2013 Héctor N. Salas. This is an open access article distributed under the Creative Commons Attribution License, which permits unrestricted use, distribution, and reproduction in any medium, provided the original work is properly cited.

\begin{abstract}
Let $X$ be a topological vector space, and let $\mathscr{B}(X)$ be the algebra of continuous linear operators on $X$. The operators $T_{1}, \ldots, T_{N} \in$ $\mathscr{B}(X)$ are disjoint hypercyclic if there is $x \in X$ such that the orbit $\left\{\left(T_{1}^{n}(x), \ldots, T_{N}^{n}(x)\right): n \in \mathbb{N}\right\}$ is dense in $X \times \ldots \times X$. Bès and Peris have shown that if $T_{1}, \ldots, T_{N}$ satisfy the Disjoint Blow-up/Collapse property, then they are disjoint hypercyclic. In a recent paper Bès, Martin, and Sanders, among other things, have characterized disjoint hypercyclic $N$-tuples of weighted shifts in terms of this property. We introduce the Strong Disjoint Blow-up/Collapse property and prove that if $T_{1}, \ldots, T_{N}$ satisfy this new property, then they have a dense linear manifold of disjoint hypercyclic vectors. This allows us to give a partial affirmative answer to one of their questions.
\end{abstract}

\section{Introduction and Background}

Let $X$ be a topological vector space, over either the real or complex numbers, whose topology has a countable basis and is complete. Let $\mathscr{B}(X)$ be the algebra of continuous linear operators on $X$.

The operator $T \in \mathscr{B}(X)$ is hypercyclic if there is $x \in X$ such that $\operatorname{Orb}(T, x):=\left\{T^{k} x: k \in \mathbb{N}\right\}$ is dense in $X$. This concept is closely related to the concept of transitivity from topological dynamics.

Definition A. The operator $T \in \mathscr{B}(X)$ is topologically transitive if for each pair $U, V$ of nonempty open subsets of $X$ there is $n$ such that $U \cap T^{-n}(V) \neq \emptyset$.

In fact, both notions are equivalent in our setting. This is the content of Birkhoff's Transitivity Theorem; see for instance 1.7 of the instructive notes by Shapiro [1].

The first version of the Hypercyclicity Criterion, whose importance is that if an operator satisfies it then it is hypercyclic, was given by Kitai in [2] and by Gethner and Shapiro in [3]. Several mathematicians had given different versions of it. One of them is the following.

Definition B. The operator $T \in \mathscr{B}(X)$ satisfies the Blowup/Collapse property if whenever nonempty open sets $W, V_{0}$,
$V_{1}$ are given with $W$ neighbourhood of 0 , then there exits $n$ such that

$$
T^{-n}(W) \cap V_{0} \neq \emptyset, \quad T^{-n}\left(V_{1}\right) \cap W \neq \emptyset .
$$

This suggestive name was coined by Grosse-Erdmann who used it in several talks that he gave years ago. The concept itself was introduced by Godefroy and Shapiro, who showed that it is implied by the Hypercyclicity Criterion [4]. BernalGonzález and Grosse-Erdmann [5] and León-Saavedra [6] showed, independently, the other implication. Thus $T$ satisfies the Blow-up/Collapse property if and only if $T$ satisfies the Hypercyclicity Criterion.

For a long time all known hypercyclic operators were known to satisfy some version of the Hypercyclicity Criterion. A milestone paper by de la Rosa and Read [7] showed that this is not always the case.

The excellent books by Bayart and Matheron [8] and Grosse-Erdmann and Peris [9] provide a solid foundation and give an overview of much of the work done in hypercyclicity. The Blow-up/Collapse property is mentioned in page 85 of [9]. The following concept was introduced independently by Bernal-González [10] and Bès and Peris [11].

Definition $C$. Let $N \geq 2$. The operators $T_{1}, \ldots, T_{N} \in \mathscr{B}(X)$ are disjoint hypercyclic if there is an $x \in X$ such that the orbit 
$\left\{\left(T_{1}^{n}(x), \ldots, T_{N}^{n}(x)\right): n \in \mathbb{N}\right\}$ is dense in $X \times \cdots \times X$. The vector $x$ is called a disjoint hypercyclic vector for $T_{1}, \ldots, T_{N}$.

It is worth noting that while the author of [10] was inspired by some recent work by Costakis and Vlachou in universal Taylor series, the authors of [11] were inspired by much older work of Furstenberg for dynamical systems in which he studied the notion of disjointness, "an extreme form of nonisomorphism" according to Parry.

The papers by Salas [12], Shkarin [13], Bès et al. [14, 15], and Bès and Martin [16] further explore different aspects of disjoint hypercyclicity.

The following three definitions were given in [11] in a slightly more general way. The first one is their Definition 2.1.

Definition $D$. Let $N \geq 2$. The operators $T_{1}, \ldots, T_{N} \in \mathscr{B}(X)$ are disjoint topologically transitive if whenever $V_{0}, V_{1}, \ldots, V_{N} \subset X$ are nonempty open sets, then there exists $n \in \mathbb{N}$ such that

$$
T_{1}^{-n}\left(V_{1}\right) \cap \cdots \cap T_{N}^{-n}\left(V_{n}\right) \cap V_{0} \neq \emptyset .
$$

The second one is included in their Proposition 2.4.

Definition $E$. Let $N \geq 2$. The operators $T_{1}, \ldots, T_{N} \in \mathscr{B}(X)$ satisfy the Disjoint Blow-up/Collapse property if for each open neighbourhood of zero $W$ and nonempty open subsets $V_{0}, V_{1}, \ldots, V_{N} \subset X$ there exists $n \in \mathbb{N}$ such that

$$
\begin{aligned}
& T_{1}^{-n}(W) \cap \cdots \cap T_{N}^{-n}(W) \cap V_{0} \neq \emptyset, \\
& T_{1}^{-n}\left(V_{1}\right) \cap \cdots \cap T_{N}^{-n}\left(V_{N}\right) \cap W \neq \emptyset .
\end{aligned}
$$

The third one is their Definition 2.5. (If $N$ were allowed to be 1 , then this would be one of the many versions of the classical Hypercyclicity Criterion.)

Definition $F$. Let $N \geq 2$. Let $\left(n_{k}\right)$ be a strictly increasing sequence of positive integers. The operators $T_{1}, \ldots, T_{N} \in$ $\mathscr{B}(X)$ satisfy the Disjoint Hypercyclicity Criterion with respect to $\left(n_{k}\right)$ provided there exist dense subsets $X_{0}, X_{1}, \ldots, X_{N}$ of $X$ and mappings $S_{l, k}: X_{l} \rightarrow X$ with $1 \leq l \leq N$ and $k \in \mathbb{N}$ satisfying

$$
\begin{aligned}
T_{l}^{n_{k}} & \longrightarrow 0 \text { pointwise on } X_{0}, \\
S_{l, k} & \longrightarrow 0 \text { pointwise on } X_{l}, \\
T_{l}^{n_{k}} S_{i, k}-\delta_{i, l} I d_{X_{i}} & \longrightarrow 0 \text { pointwise on } X_{i} \text { with } 1 \leq i \leq N .
\end{aligned}
$$

In the last display $I d_{X_{i}}$ means the identity in $X_{i}$. (It has to be $X_{i}$ and not $X_{l}$ because $X_{i}$ is the domain of $S_{i, k}$.) We now give some known relations between all these concepts.

Proposition 2.3 of [11] says that $T_{1}, \ldots, T_{N} \in \mathscr{B}(X)$ are disjoint topologically transitive if and only if the set of disjoint hypercyclic vectors for $T_{1}, \ldots, T_{N}$ is a dense $G_{\delta}$ of $X$.

Proposition 2.4 of [11] says that if $T_{1}, \ldots, T_{N} \in \mathscr{B}(X)$ satisfy the Disjoint Blow-up/Collapse property, then they are disjoint topologically transitive. This is the disjoint version of Theorem 1.2 of [4], but the authors of [4] assume that $X$ is a Banach space.
Theorem 2.7 of [11] says that $T_{1}, \ldots, T_{N} \in \mathscr{B}(X)$ satisfy the Disjoint Hypercyclicity Criterion if and only if for each $r \in \mathbb{N}$ the operators $\overbrace{T_{1}, \ldots, T_{1}}^{r}, \ldots, \overbrace{T_{N}, \ldots, T_{N}}^{r}$ are disjoint topologically transitive in $\mathscr{B}\left(X^{r}\right)$.

Two comments are in order.

(a) Theorem 2.7 of [11] also gives another equivalence.

(b) When $N=1$, Theorem 2.7 of [11] is another equivalence of the Hypercyclicity Criterion. This equivalence is given by the same authors in an earlier paper, Theorem 2.3 of [17].

Assume $2 \leq N$. Bès et al. show in Theorems 2.1 and 2.2 of [18] that $N$ weighted shifts are disjoint hypercyclic if and only if they satisfy the Disjoint Blow-up/Collapse property. They also show that $N$ weighted shifts can never satisfy the Disjoint Hypercyclicity Criterion; see Proposition 3.2 in [18]. The relative simplicity with which the authors of [18] show disjoint hypercyclic operators which do not satisfy the Disjoint Hypercyclicity Criterion should be contrasted with the sophistication of the arguments in [7].

The authors of [18] also point out that if $T_{1}, \ldots, T_{N} \in$ $\mathscr{B}(X)$ satisfy the Disjoint Hypercyclicity Criterion, then $T_{1}, \ldots, T_{N}$ satisfy the Disjoint Blow-up/Collapse property; see their last diagram.

It is our goal in this paper to study a "strong" version of the Blow-up/Collapse property. We show that for the class of $N$-tuples of weighted shifts the Disjoint Blow-up/Collapse property and its strong version are equivalent. Our main result is that if $T_{1}, \ldots, T_{N}$ satisfy the Strong Disjoint Blowup/Collapse property, then they have a dense linear manifold of disjoint hypercyclic vectors. We conclude the paper with some open questions.

\section{Preliminary Results}

For convenience, in all what follows, the open neighbourhoods of zero will be chosen to be balanced; that is, $W=$ $\cup_{|\lambda| \leq 1} \lambda W$.

Definition 1. The operators $T_{1}, \ldots, T_{N} \in \mathscr{B}(X)$ are said to satisfy the Strong Disjoint Blow-up/Collapse property if for all $m \geq 1$ whenever nonempty open sets $W, V_{1-m}, \ldots, V_{0}, V_{1}, \ldots, V_{N}$ are given with $W$ a neighborhood of 0 , then there exists $n$ such that

$$
\begin{gathered}
T_{1}^{-n}(W) \cap \cdots \cap T_{N}^{-n}(W) \cap V_{k-m} \neq \emptyset \quad \text { for } 1 \leq k \leq m, \\
T_{1}^{-n}\left(V_{1}\right) \cap \cdots \cap T_{N}^{-n}\left(V_{N}\right) \cap W \neq \emptyset .
\end{gathered}
$$

Remark 2. (a) The Disjoint Blow-up/Collapse property, Definition E, results when $m$ is only allowed to be 1 .

(b) If $T$ satisfies the Blow-up/Collapse property, then by using that $T$ satisfies the Hypercyclicity Criterion (see paragraph after Definition B) one can prove that $T$ also satisfies the Strong Blow-up/Collapse property.

Proposition 2.4 of [11], which was stated without proof, results from the following proposition when $U=V_{0}, m=1$, and the word "strong" is omitted. 
Proposition 3. Let $T_{1}, \ldots, T_{N} \in \mathscr{B}(X)$ satisfy the Strong Disjoint Blow-up/Collapse property. If $m \geq 1$ and the nonempty open sets $W, U, V_{1}, \ldots, V_{N}$ and $V_{1-m}, \ldots, V_{0}$ are given with $W$ a neighborhood of 0 , then there exists $n$ such that (5) holds and

$$
T_{1}^{-n}\left(V_{1}\right) \cap \cdots \cap T_{N}^{-n}\left(V_{N}\right) \cap U \neq \emptyset .
$$

Proof. Let $x_{j} \in V_{j}$ for $1 \leq j \leq N$ and $x \in U$. Let $W^{\prime}$ be an open set containing 0 such that $W^{\prime}+W^{\prime} \subset W$ and $x_{j}+W^{\prime}+$ $W^{\prime} \subset V_{j}$ for $1 \leq j \leq N$ and $x+W^{\prime}+W^{\prime} \subset U$.

Set $V_{-m}^{\prime}=x+W^{\prime}$ and $V_{i}^{\prime}=V_{i}$ for $1-m \leq i \leq 0$ and $V_{j}^{\prime}=x_{j}+W^{\prime}$ for $1 \leq j \leq N$. Since $T_{1}, \ldots, T_{N} \in \mathscr{B}(X)$ satisfy the Strong Disjoint Blow-up/Collapse property, we have that there exist $n \in \mathbb{N}$ and $z_{i} \in V_{i}^{\prime}$ for $-m \leq i \leq 0$ and $w \in W^{\prime}$ such that $T_{j}^{n}\left(z_{i}\right) \in W^{\prime}$ and $T_{j}^{n}(w) \in V_{j}^{\prime}$ for $-m \leq i \leq 0$ and $1 \leq j \leq N$. It remains to check that

$$
T_{1}^{-n}\left(V_{1}\right) \cap \cdots \cap T_{N}^{-n}\left(V_{N}\right) \cap U \neq \emptyset .
$$

Let $z=z_{-m}+w \in x+W^{\prime}+W^{\prime} \subset U$ and $T_{j}^{n}(z) \in V_{j}^{\prime}+W^{\prime} \subset V_{j}$ for $1 \leq j \leq N$. Then $z \in T_{1}^{-n}\left(V_{1}\right) \cap \cdots \cap T_{N}^{-n}\left(V_{N}\right) \cap U$.

As indicated in the introduction, it was pointed out in [18] that the following proposition is true when the word "strong" is eliminated.

Proposition 4. If $T_{1}, \ldots, T_{N} \in \mathscr{B}(X)$ satisfy the Disjoint Hypercyclicity Criterion, then they also satisfy the Strong Disjoint Blow-up/Collapse property.

Proof. Let $W$ be an open neighbourhood of zero and $V_{1-m}, \ldots, V_{0}, V_{1}, \ldots, V_{N} \subset X$ be nonempty open sets. Assume that $X_{0}, X_{1}, \ldots, X_{N}$ are the dense subsets of $X$ and $S_{l, k}$ : $X_{l} \rightarrow X$ with $1 \leq l \leq N$ the mappings given by the Disjoint Hypercyclic Criterion. Let $z_{j} \in V_{j} \cap X_{0}$ for $1-m \leq j \leq 0$ and $y_{i} \in V_{i} \cap X_{i}$ for $1 \leq i \leq N$. We now choose another open neighbourhood of zero $W^{\prime}$ with $W^{\prime} \subset W$ and such that $1 \leq i \leq N$ and

$$
\overbrace{W^{\prime}+\cdots+W^{\prime}}^{N} \subset W, \quad y_{i}+\overbrace{W^{\prime}+\cdots+W^{\prime}}^{N} \subset V_{i} .
$$

By hypothesis there exists $n_{k}$ so that $T_{i}^{n_{k}}\left(z_{j}\right) \in W^{\prime}$, for all $1 \leq$ $i \leq N$ and $1-m \leq j \leq 0$, and, for $1 \leq l \leq N$,

$$
S_{l, k}\left(y_{l}\right) \in W^{\prime}, \quad T_{l}^{n_{k}} S_{i, k}\left(y_{i}\right)-\delta_{l, i} y_{i} \subset W^{\prime} .
$$

Thus (5) is satisfied. It remains to verify that (6) is also satisfied. For that we choose $w_{0}=\sum_{i=1}^{N} S_{i, k}\left(y_{i}\right)$ and it follows that

$$
w_{0} \in T_{1}^{-n_{k}}\left(V_{1}\right) \cap \cdots \cap T_{N}^{-n_{k}}\left(V_{N}\right) \cap W .
$$

The following proposition has an immediate proof, and it is often used when studying weighted shifts. Let $\mathbb{K}$ be either $\mathbb{N}$ or $\mathbb{Z}$, and let $X$ be $l^{p}(\mathbb{K})$ for $1 \leq p<\infty$ or $c_{0}(\mathbb{K})$. Let $\left\{e_{i}: i \in \mathbb{K}\right\}$ be the canonical basis of $X$ and for $x \in X$ we denote by $\left\langle x, e_{i}^{*}\right\rangle=e_{i}^{*}(x)$, where $e_{i}^{*} \in X^{*}$ and $e_{i}^{*}\left(e_{j}\right)=\delta_{i, j}$. We say that the vector $y$ dominates the vector $x$ if

$$
\left|\left\langle x, e_{i}\right\rangle\right| \leq\left|\left\langle y, e_{i}\right\rangle\right| \quad \forall i .
$$

Proposition 5. Let $T$ be a weighted shift. If the vector a dominates the vector $b$, then for all $n \in \mathbb{N}$,

$$
\left\|T^{n}(b)\right\| \leq\left\|T^{n}(a)\right\| .
$$

The following result says that for the class of $N$-tuples of weighted shifts the Disjoint Blow-up/Collapse property and its strong version coincide.

Proposition 6. Let $X$ be $l^{p}(\mathbb{K})$ for $1 \leq p<\infty$ or $c_{0}(\mathbb{K})$. If $T_{1}, \ldots, T_{N}$ are disjoint hypercyclic weighted shifts, then they satisfy the Strong Disjoint Blow-up/Collapse property.

Proof. By Theorems 2.1 and 2.2 of [18] these operators satisfy the Disjoint Blow-up/Collapse property. We have to prove that this implies that the strong version is also satisfied.

We prove it for $N=2$ and for $l^{2}(\mathbb{N})$ or $l^{2}(\mathbb{Z})$ which illustrate the general method. Let $W=\{x:\|x\|<\delta\}$ and $V_{2}, V_{1}, V_{0}, \ldots, V_{-k}$ for some $k \in \mathbb{N}$. Let $\left\{e_{i}: i \in \mathbb{N}\right\}$ and $\left\{e_{i}:\right.$ $i \in \mathbb{Z}\}$ be the orthonormal canonical basis, respectively, with respect to which both operators are weighted shifts. We can assume without loss of generality that there exist $x_{0}, \ldots, x_{-k}$ in the span of $\left\{e_{i}: i \in A\right\}$ such that $\left\{x:\left\|x-x_{j}\right\|<\epsilon\right\} \subset V_{-j}$ for $j=0, \ldots, k$ and $A$ is a finite interval of either $\mathbb{N}$ or $\mathbb{Z}$ and $\epsilon<1 / 2$. Let $M=\max \left\{\left\|x_{-j}\right\|+1: j=0, \ldots, k\right\}$. Let us choose $z_{0}$ such that $\left\langle z, e_{i}\right\rangle=M$ if $i \in A$ and 0 otherwise. Set $\widehat{V}_{0}=\{x:\|x-z\|<\epsilon\}$. Apply the Disjoint Blow-up/Collapse property to $W$ and $V_{2}, V_{1}$, and $\widehat{V}_{0}$. This means that there is an arbitrarily large $n$ such that

$$
\begin{aligned}
& \widehat{V}_{0} \cap T_{1}^{-n}(W) \cap T_{2}^{-n}(W) \neq \emptyset, \\
& W \cap T_{1}^{-n}\left(V_{1}\right) \cap T_{2}^{-n}\left(V_{2}\right) \neq \emptyset .
\end{aligned}
$$

Case $1\left(l^{2}(\mathbb{N})\right)$. If $n$ is large enough, we have that $T_{1}^{n}(x)=0=$ $T_{2}^{n}(x)$ for all $x$ in the span of $\left\{e_{i}: i \in A\right\}$, in particular for $x_{0}, \ldots, x_{-k}$. Thus

$$
V_{-j} \cap T_{1}^{-n}(W) \cap T_{2}^{-n}(W) \neq \emptyset
$$

for $j=0, \ldots, k$.

Case $2\left(l^{2}(\mathbb{Z})\right)$. There exists $z \in \widehat{V}_{0}$ such that $\left\|T_{1}^{n}(z)\right\|<\delta$ and $\left\|T_{2}^{n}(z)\right\|<\delta$. Since $\epsilon<1 / 2$ and $\left\|e_{i}\right\|=1$, we have for $j=0, \ldots, k$ the following:

$$
\begin{aligned}
\left|\left\langle z, e_{i}\right\rangle\right| & \geq\left|\left\langle z_{0}, e_{i}\right\rangle\right|-\left\|\left(z-z_{0}\right)\right\| \\
& \geq M-\frac{1}{2}>\left\|x_{-j}\right\| \geq\left|\left\langle x_{-j}, e_{i}\right\rangle\right|
\end{aligned}
$$

for $i \in A$; but for $i \notin A,\left|\left\langle z, e_{i}\right\rangle\right| \geq 0=\left|\left\langle x_{-j}, e_{i}\right\rangle\right|$. Thus $x_{0}, \ldots, x_{-k}$ are dominated by $z$, and we are done. 
Corollary 7. The converse of Proposition 4 is not true.

Proof. By Theorems 2.1 and 2.2 of [18] disjoint hypercyclic weighted shifts satisfy the Disjoint Blow-up/Collapse property but by Proposition 2.3 of [18] cannot satisfy the Disjoint Hypercyclicity Criterion. By using Proposition 6 we can conclude the proof of the corollary.

If $T \in \mathscr{B}(X)$ is hypercylic, Herrero [19] and Bourdon [20], independently, showed that $T$ has a dense linear manifold of hypercyclic vectors. (See also page 53 of [9].) If $T_{1}, \ldots, T_{N}$ are disjoint hypercyclic, it is not known whether their set of disjoint hypercyclic vectors is dense in $X$, page 115 of [10]. In view of the above results, the authors of [18] pose their Problem 3.6 which is the following.

Problem $G$. Let $T_{1}, \ldots, T_{N}$ be densely disjoint hypercyclic operators in $\mathscr{B}(X)$. Must they support a dense disjoint hypercyclic manifold?

In the following section we give a partial affirmative answer whenever $T_{1}, \ldots, T_{N}$ satisfy the Strong Disjoint Blowup/Collapse property.

\section{Main Result}

In the theorem below, it is worth noting that it is not necessary to suppose beforehand that the operators have a dense set of disjoint hypercyclic vectors; this follows from the construction. However, since $T_{1}, \ldots, T_{N} \in \mathscr{B}(X)$ satisfy the Disjoint Blow-up/Collapse property, they have a dense set of disjoint hypercyclic vectors which is a $G_{\delta}$, Proposition 2.3 of [11].

Theorem 8. Let $X$ be a topological vector space, over either $\mathbb{R}$ or $\mathbb{C}$, whose topology has a countable basis and is complete. Let $T_{1}, \ldots, T_{N} \in \mathscr{B}(X)$ satisfy the Strong Disjoint Blowup/Collapse property. Then $T_{1}, \ldots, T_{N}$ have a dense linear manifold of disjoint hypercyclic vectors.

Proof. We prove the theorem when $N=2$. The proof for an arbitrary $N$ is conceptually the same, but the notation is more cumbersome.

The setting up of the proof is as follows. For each $i$ we find a sequence $\{x(i, k, j)\}$ with $1 \leq j \leq i+k$ and such that the order in which the vectors are generated is the lexicographic order for $(k, j)$. The limit for $k \rightarrow \infty$ of $x(i, k, j)=x_{i}$ will exist. The linear manifold of disjoint hypercyclic vectors is the span of the $x_{i}^{\prime} s$.

Let $\left\{\left(y_{k, 1}, y_{k, 2}\right): k \in \mathbb{N}, u=1,2\right\}$ be dense in $X \times X$, and let $\left\{z_{p}: p \in \mathbb{N}\right\}$ be dense in $X$. Let $A_{1} \cup A_{2} \cup A_{3} \cup \cdots=\mathbb{N}$ be a partition of $\mathbb{N}$ such that each $A_{p}$ is an infinite set and define $f_{j}=z_{p}$ whenever $j \in A_{p}$.

Let $\left\{G_{n}: n \in \mathbb{N}\right\}$ be a local basis of 0 such that each $G_{n}$ is a balanced open set and $\overline{G_{n+1}} \varsubsetneqq G_{n}$.

We now proceed to the construction of the vectors $\{x(i, k, j)\}$. In each step after the third one, we find several vectors at the same time thanks to the strong version of the Disjoint Blow-up/Collapse property, Definition 1. (We use properties (5) and (7).)
Step $n$ corresponds to the $(i, k)$ for which $n=(i+k-1)(i+$ $k) / 2+1-i$. In this step $i+k-1$ vectors are found.

We choose $x(i, 1,1) \in f_{i}+G_{i}$ for all $i \in \mathbb{N}$, and, moreover, $x(i, k, j) \in f_{i}+G_{i}$ for all $k$ and $1 \leq j \leq i+k$. We also have that their limit $x_{i} \in f_{i}+G_{i}$. In this way we ensure that $\left\{x_{i}: i \in \mathbb{N}\right\}$ is dense in $X$ since for $j \in A_{p}$ we have that $\lim _{j \rightarrow \infty} x_{j}=z_{p}$.

Step 1. Let $U=f_{1}+G_{1}$ and $V_{u}=y_{1, u}+G_{1}$ for $u=1,2$. Then by (7) we have $x(1,1,1) \in U$ and $m_{1}$ such that for $u=1,2$

$$
T_{u}^{m_{1}}(x(1,1,1)) \in V_{u} .
$$

Since $T_{1}, T_{2}$ are continuous, there exists $W_{2}=G_{p_{2}}$ such that $1=p_{1}<p_{2}$ and $x-x(1,1,1) \in \bar{W}_{2}$ implies that $T_{u}^{m_{1}}(x) \in$ $V_{u}$ for $u=1,2$. In addition, since $\left\{G_{k}\right\}$ is a local basis, we can choose $W_{2}$ sufficiently small that $x(1,1,1)+\bar{W}_{2} \subset f_{1}+G_{1}$.

Step 2. Let $U=f_{2}+G_{2}$ and $V_{u}=y_{2, u}+G_{2}$ for $u=1,2$. Let $V_{0}=x(1,1,1)+W_{2}$ and $W=W_{2}$. By applying (5) and (7) we find $x(2,1,1) \in U$ and $x(1,1,2) \in V_{0}$ and $m_{2}$ such that for $u=1,2$

$$
T_{u}^{m_{2}}(x(2,1,1)) \in V_{u}, \quad T_{u}^{m_{2}}(x(1,1,2)) \in W_{2} .
$$

Let $W_{3}=G_{p_{3}}$ be such that $p_{2}<p_{3}$ and $x-x(2,1,1) \in \bar{W}_{3}$ implies that $T_{u}^{m_{2}}(x)-y_{2, u} \in W_{2}$, and if $x-x(1,1,2) \in \bar{W}_{3}$, then $T_{u}^{m_{2}}(x) \in W_{2}$. Also we can choose $W_{3}$ such that $x(1,1,2)+$ $\bar{W}_{3} \subset V_{0}$.

Step $n$. Let $(i, k)$ be such that $n=(i+k-1)(i+k) / 2+1-i$.

The open set $W_{n}=G_{p_{n}}$ has been chosen in the previous step. Let

$$
V_{u}=y_{n, u}+G_{n} \quad \text { for } u=1,2
$$

Case $(k=1)$. We have that $1<i$ since $3 \leq n$. Set $U=f_{i}+G_{i}$ and for $0 \leq t \leq i-2$ let

$$
x(i-1-t, 1+t, i-1-t)+W_{n}=V_{-t} .
$$

Case $(i=1)$. Set $U=x(1, k-1, k)+W_{n}$ and for $2 \leq s \leq k$ let

$$
x(s, k+1-s, s-1)+W_{n}=V_{2-s} .
$$

Case $(1<k, 1<i)$. Set $U=x(i, k-1, i+k-1)+W_{n}$ and for $0 \leq t \leq i-2$ let

$$
x(i-1-t, k+t, k+i-2-t)+W_{n}=V_{-t}
$$

and for $2 \leq s \leq k$ let

$$
x(i+s-1, k+1-s, s-1)+W_{n}=V_{3-i-s} .
$$

Setting $W=W_{n}$ and using (5) and (7) there are $k+i-1$ vectors and $m_{n}$ such that $x(i, k, 1) \in U$ satisfy, for $u=1,2$,

$$
T_{u}^{m_{n}}(x(i, k, 1)) \in V_{u} .
$$


And the remaining vectors chosen in this step satisfy the following displayed formulas. (Clearly when $i=1$ only (28) matters, whereas when $k=1$ only (29) matters.)

For $0 \leq t \leq i-2$ we have that $x(i-1-t, k+t, k+i-1-t) \in V_{-t}$

$$
\begin{array}{r}
T_{u}^{m_{n}}(x(i-1-t, k+t, k+i-1-t)) \in W_{n} \\
\text { for } 0 \leq t \leq i-2 .
\end{array}
$$

For $2 \leq s \leq k$ we have $x(i+s-1, k+1-s, s) \in V_{3-i-s}$ and

$$
T_{u}^{m_{n}}(x(i+s-1, k+1-s, s)) \in W_{n} \quad \text { for } 2 \leq s \leq k .
$$

We now choose a sufficiently small open set $W_{n+1}=G_{p_{n+1}}$ with $p_{n}<p_{n+1}$ and such that $x-x(i, k, 1) \in \bar{W}_{n+1}$ implies that

$$
T_{u}^{m_{n}}(x) \in y_{n, u}+G_{n},
$$

and for the other vectors $x(c, a, b)$ with $c \neq i$ chosen at this stage we also have that $x-x(c, a, b) \in \bar{W}_{n+1}$ implies that

$$
T_{u}^{m_{n}}(x) \in W_{n}
$$

for $u=1,2$. Moreover, we also have that for all vectors $x(c, a, b)$ chosen at this stage

$$
x(c, a, b)+\bar{W}_{n+1} \subset x\left(c, a_{-}, b_{-}\right)+W_{n},
$$

where $\left(a_{-}, b_{-}\right)$is the immediate predecessor of $(a, b)$ in the lexicographic order. When $a=1=b$ we have that

$$
x(i, 1,1)+\bar{W}_{n+1} \subset f_{n}+G_{n} .
$$

Since $\left\{W_{n}: n \in \mathbb{N}\right\}$ is also a local basis of 0 and $X$ is complete, it follows from (29) and (30) that for each $i$ the sequence $\{x(i, k, j)\}$ converges to a vector $x_{i}$. Moreover, (27) and (28) and $n=(k+i-1)(k+i) / 2+1-i$ imply that

$$
T_{u}^{m_{n}}\left(x_{i}\right)-y_{n, u} \in W_{n}, \quad T_{u}^{m_{n}}\left(x_{q}\right) \in W_{n}
$$

for $q \neq i$.

To finish the proof we have to prove that if $x=\sum_{i=1}^{M} \lambda_{i} x_{i}$ with some $\lambda_{i} \neq 0$, then $x$ is disjoint hypercyclic. To that end choose $\lambda_{i_{0}}$ such that $\left|\lambda_{i_{0}}\right| \geq \lambda_{i}$ for $i=1, \ldots, M$. Since nonzero multiples of disjoint hypercyclic vectors are also disjoint hypercyclic, we may assume that $\lambda_{i_{0}}=1$. For $M<k$ and $n=\left(i_{0}+k-1\right)\left(i_{0}+k\right) / 2+1-i_{0}$ we have that $x_{i_{0}} \in$ $x\left(i_{0}, k, 1\right)+\bar{W}_{n+1}$ and for $i \neq i_{0}$ and $i \leq M$ we have that $x_{i} \in x(i, a, b)+\bar{W}_{n+1}$, where the vector $x(i, a, b)$ is obtained in the $n$ step. Therefore for $u=1,2$ we use (31) to get

$$
T_{u}^{m_{n}}\left(x_{i_{0}}+\sum_{i \neq i_{0}, i \leq M} \lambda_{i} x_{i}\right)-y_{n, u} \in G_{n}+\overbrace{W_{n}+W_{n}+\cdots W_{n}}^{M-1}
$$

since $\lambda_{i} \leq 1$ for $i \neq i_{0}$ and the open sets have been chosen to be balanced. Thus we have shown that $x$ is a disjoint hypercyclic vector.
The authors of [6] comment that they do not know the answer to Problem 3.6 of [6] (Problem G) even when the operators are weighted shifts.

Corollary 9. Let $X$ be $l^{p}(\mathbb{K})$ for $1 \leq p<\infty$ or $c_{0}(\mathbb{K})$. If $T_{1}, \ldots, T_{N}$ are disjoint hypercyclic weighted shifts, then they have a dense linear manifold of disjoint hypercyclic vectors.

Proof. Proposition 6 and Theorem 8 provide the proof.

\section{Concluding Questions}

Among the following questions the most fundamental is the first one. The next three questions might be easier to handle for the class of $N$-tuples of weighted shifts thanks to the results of [18] which also characterize disjoint hypercyclicity in terms of their weight sequence.

First we recall the relevant definitions. A hypercyclic subspace for $T \in \mathscr{B}(X)$ is an infinite dimensional subspace whose nonzero vectors are hypercyclic. The systematic study of hypercyclic subspaces started with work by BernalGonzález and Montes-Rodríguez in [21]. Chapter 8 of [8] and Chapter 10 of [9] give the fundamentals and history of hypercyclic subspaces.

The operator $T \in \mathscr{B}(X)$ is supercyclic if there is an $x \in X$ such that $\left\{\lambda T^{k} x: k \in \mathbb{N}, \lambda\right.$ scalar $\}$ is dense in $X$. Chapter 9 of [8] treats supercyclicity. A supercyclic subspace for $T \in \mathscr{B}(X)$ is an infinite dimensional subspace whose nonzero vectors are supercyclic. A panorama of supercyclic subspaces is given by Montes-Rodríguez and Salas in their survey [22].

A disjoint hypercyclic subspace for $T_{1}, \ldots, T_{N} \in \mathscr{B}(X)$ is an infinite dimensional subspace whose nonzero vectors are disjoint hypercyclic. Proposition 3.7 of [18] assures the existence of disjoint hypercyclic subspaces in some cases. Another line of inquiry is to study disjoint supercyclicity; see [14-16]. Montes-Rodríguez and Salas characterized supercyclic subspaces for the class of weighted shifts [23]; see also [22].

Question 1. Are the Disjoint Blow-up/Collapse property and its strong version equivalent?

Question 2. Given $T_{1}, \ldots, T_{N} \in \mathscr{B}(X)$ that satisfy the Disjoint Blow-up/Collapse property, can we add some $T_{N+1}$ such that the resultant $N+1$-tuple still satisfies the Disjoint Blow-up/Collapse property?

Question 3. Given $T_{1}, \ldots, T_{N} \in \mathscr{B}(X)$ which are disjoint hypercyclic, can we add some $T_{N+1}$ such that the resultant $N+1$-tuple is still disjoint hypercyclic?

Question 4. Assume that $T_{1}, \ldots, T_{N} \in \mathscr{B}(X)$ have a disjoint hypercyclic space. Can we add some $T_{N+1}$ such that the resultant $N+1$-tuple still has a disjoint hypercyclic subspace?

In the last three questions $X$ is either $l^{p}(\mathbb{K})$ for $1 \leq p<\infty$ or $c_{0}(\mathbb{K})$.

Question 5. Which weighted shifts $T_{1}, \ldots, T_{N} \in \mathscr{B}(X)$ have a disjoint hypercyclic space? 
Question 6. Which weighted shifts $T_{1}, \ldots, T_{N} \in \mathscr{B}(X)$ are disjoint supercyclic?

Question 7. Which weighted shifts $T_{1}, \ldots, T_{N} \in \mathscr{B}(X)$ have a disjoint supercyclic space?

More unsolved questions on disjoint hypercyclicity can be found in $[10-12,14,15,18]$. Unsolved questions in supercyclicity can be found in $[22,23]$.

\section{Acknowledgment}

The author would like to thank the referees for their very useful comments. I would also like to thank Professor Dorothy Bollman for her invaluable insights.

\section{References}

[1] J. H. Shapiro, "Notes on the dynamics of linear operators," http://www.math.msu.edu/ shapiro/Pubvit/Downloads/ LinDynamics/lindynamics.pdf.

[2] C. Kitai, Invariant closed sets for linear operators [Ph.D. thesis], University of Toronto, 1982.

[3] R. M. Gethner and J. H. Shapiro, "Universal vectors for operators on spaces of holomorphic functions," Proceedings of the American Mathematical Society, vol. 100, no. 2, pp. 281-288, 1987.

[4] G. Godefroy and J. H. Shapiro, "Operators with dense, invariant, cyclic vector manifolds," Journal of Functional Analysis, vol. 98, no. 2, pp. 229-269, 1991.

[5] L. Bernal-González and K.-G. Grosse-Erdmann, "The hypercyclicity criterion for sequences of operators," Studia Mathematica, vol. 157, no. 1, pp. 17-32, 2003.

[6] F. León-Saavedra, "Notes about the hypercyclicity criterion," Mathematica Slovaca, vol. 53, no. 3, pp. 313-319, 2003.

[7] M. de la Rosa and C. J. Read, "A hypercyclic operator whose direct sum $T \oplus T$ is not hypercyclic," Journal of Operator Theory, vol. 61, no. 2, pp. 369-380, 2009.

[8] F. Bayart and E. Matheron, Dynamics of linear operators, vol. 179 of Cambridge Tracts in Mathematics, Cambridge University Press, Cambridge, UK, 2009.

[9] K.-G. Grosse-Erdmann and A. Peris Manguillot, Linear Chaos, Universitext, Springer, London, UK, 2011.

[10] L. Bernal-González, "Disjoint hypercyclic operators," Studia Mathematica, vol. 182, no. 2, pp. 113-131, 2007.

[11] J. Bès and A. Peris, "Disjointness in hypercyclicity," Journal of Mathematical Analysis and Applications, vol. 336, no. 1, pp. 297315, 2007.

[12] H. N. Salas, "Dual disjoint hypercyclic operators," Journal of Mathematical Analysis and Applications, vol. 374, no. 1, pp. 106117, 2011.

[13] S. Shkarin, "A short proof of existence of disjoint hypercyclic operators," Journal of Mathematical Analysis and Applications, vol. 367, no. 2, pp. 713-715, 2010.

[14] J. Bès, Ö. Martin, and A. Peris, "Disjoint hypercyclic linear fractional composition operators," Journal of Mathematical Analysis and Applications, vol. 381, no. 2, pp. 843-856, 2011.

[15] J. Bès, Ö. Martin, A. Peris, and S. Shkarin, "Disjoint mixing operators," Journal of Functional Analysis, vol. 263, no. 5, pp. 1283-1322, 2012.
[16] J. Bès and Ö. Martin, "Compositional disjoint hypercyclicity equals disjoint supercyclicity," Houston Journal of Mathematics, vol. 38, no. 4, pp. 1149-1163, 2012.

[17] J. Bès and A. Peris, "Hereditarily hypercyclic operators," Journal of Functional Analysis, vol. 167, no. 1, pp. 94-112, 1999.

[18] J. Bès, Ö. Martin, and R. Sanders, "Weighted shifts and disjoint hypercyclicity," submitted to Journal of Operator Theory. In press.

[19] D. A. Herrero, "Limits of hypercyclic and supercyclic operators," Journal of Functional Analysis, vol. 99, no. 1, pp. 179-190, 1991.

[20] P. S. Bourdon, "Invariant manifolds of hypercyclic vectors," Proceedings of the American Mathematical Society, vol. 118, no. 3, pp. 845-847, 1993.

[21] L. Bernal-González and A. Montes-Rodríguez, "Non-finitedimensional closed vector spaces of universal functions for composition operators," Journal of Approximation Theory, vol. 82, no. 3, pp. 375-391, 1995.

[22] A. Montes-Rodríguez and H. N. Salas, "Supercyclic subspaces," The Bulletin of the London Mathematical Society, vol. 35, no. 6, pp. 721-737, 2003.

[23] A. Montes-Rodríguez and H. N. Salas, "Supercyclic subspaces: spectral theory and weighted shifts," Advances in Mathematics, vol. 163, no. 1, pp. 74-134, 2001. 


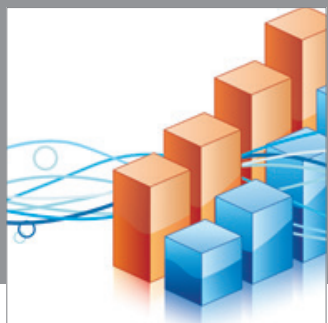

Advances in

Operations Research

mansans

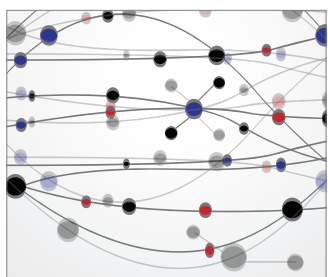

The Scientific World Journal
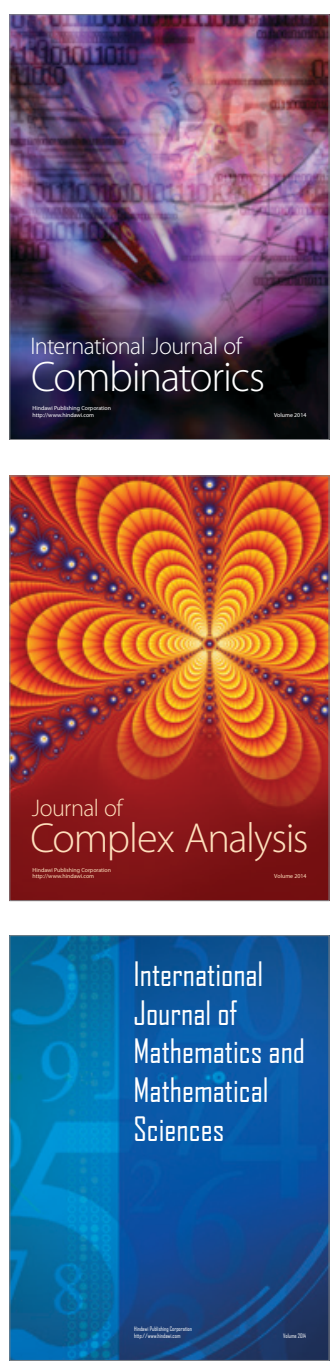
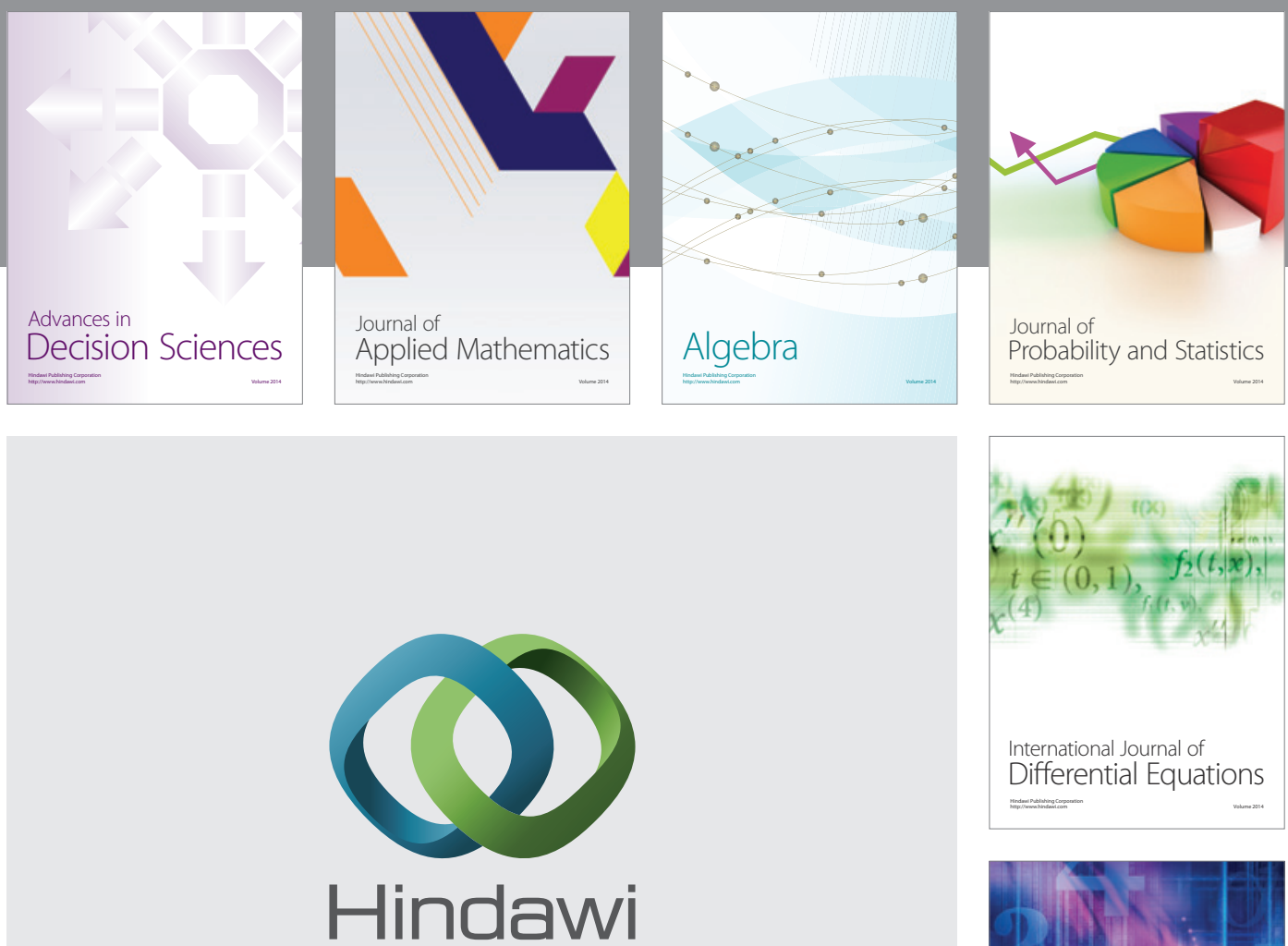

Submit your manuscripts at http://www.hindawi.com
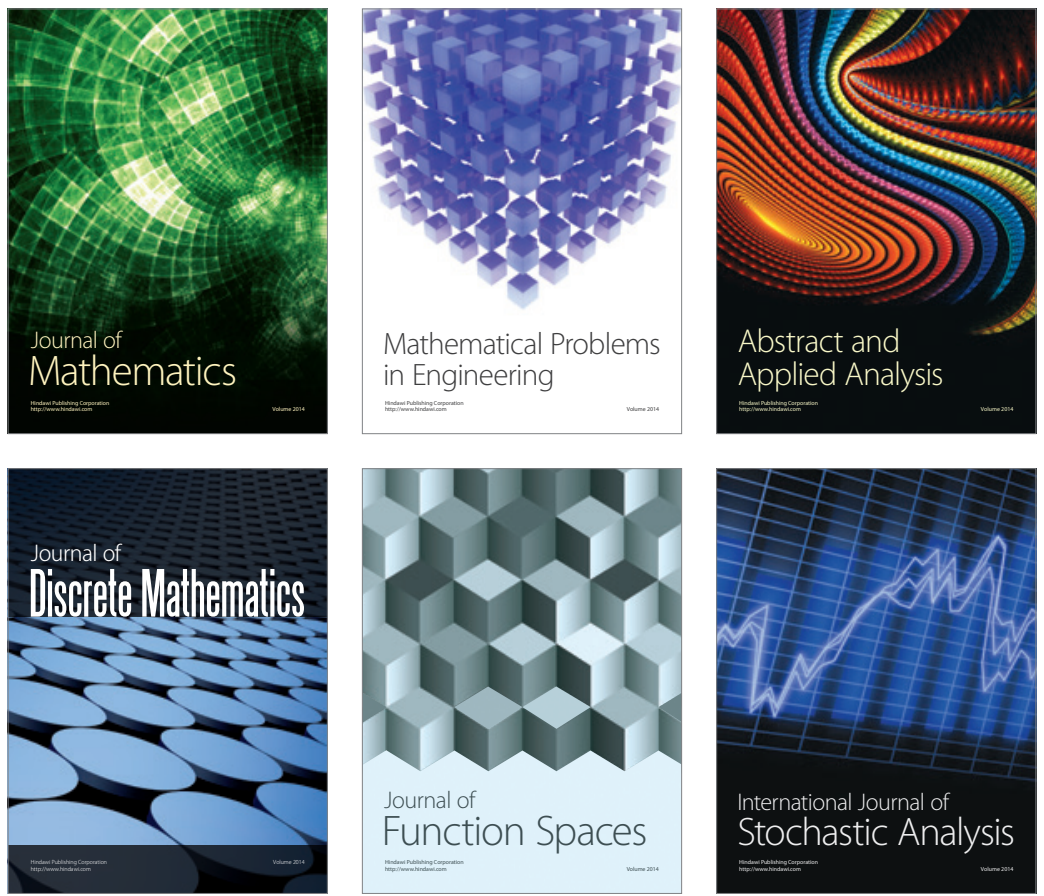

Journal of

Function Spaces

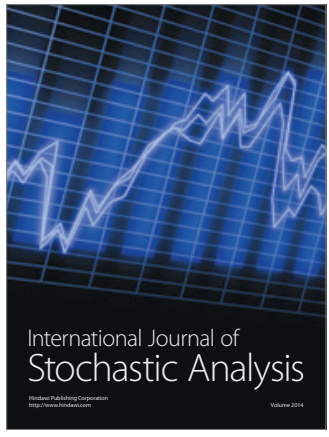

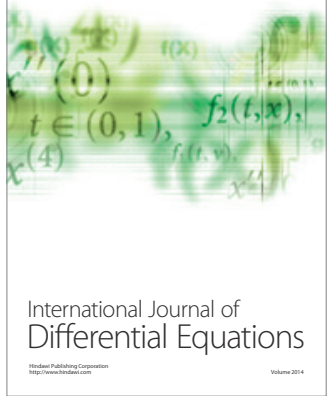
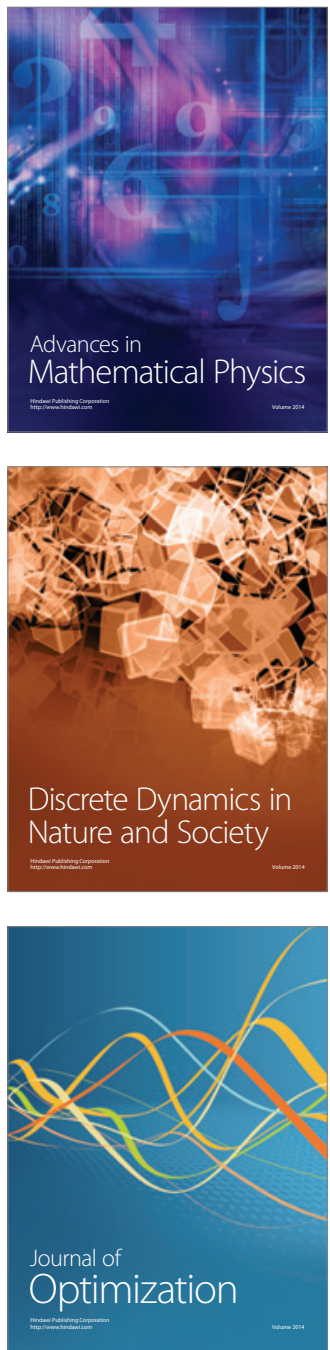